\title{
Isolation of Non-Hydrogen Sulfide-Producing Salmonella enterica Serovar Infantis from a Clinical Sample: the First Case in Korea
}

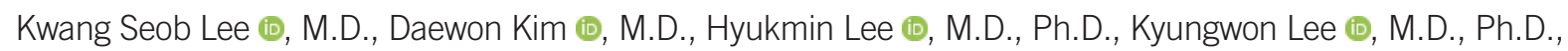 \\ and Dongeun Yong (i), M.D., Ph.D. \\ Department of Laboratory Medicine and Research Institute of Bacterial Resistance, Yonsei University College of Medicine, Seoul, Korea
}

\section{Dear Editor,}

Salmonella is one of the major pathogens causing bacterial gastroenteritis or foodborne disease worldwide. Infections with nontyphoidal Salmonella have been reported in more than 2,000 patients in Korea every year from 2017 to 2019, and it is the fifth most common cause of gastrointestinal infections in Korea following norovirus, C. perfringens, Campylobacter, rotavirus group A [1]. Non-typhoidal Salmonella infection usually manifests as acute onset of fever, chills, vomiting, and diarrhea, and focal or blood-stream infections develop in some cases [2].

Production of hydrogen sulfide $\left(\mathrm{H}_{2} \mathrm{~S}\right)$ is a unique property of Salmonella, and it is commonly detected to differentiate Salmonella species from other bacterial pathogens using biochemical test media such as triple sugar iron (TSI) agar. However, several non- $\mathrm{H}_{2} \mathrm{~S}$-producing Salmonella species have been isolated in various countries (mainly China) from poultry meat [3] or clinical samples such as stool or sacral wound (Table 1) [4-10]. Enzymes encoded by the phs, cysJIH, and asr operons are essential for $\mathrm{H}_{2} \mathrm{~S}$ production. Previous studies reported nonsense mu- tations at different sites on the phs operon in $\mathrm{H}_{2} \mathrm{~S}$-negative Salmonella strains [8]. Here, we report a non- $\mathrm{H}_{2} \mathrm{~S}$-producing Salmonella enterica serovar Infantis clinical isolate obtained from the blood sample of a Korean patient, the first such finding in Korea to our knowledge (Table 1). This study was approved by the Institutional Review Board.

A 63-year-old woman was admitted to Severance Hospital, Seoul, Korea, from January to February 2019 for flank and pelvic pain, and poor general condition. Eight years ago, she was diagnosed as having pancreatic neuroendocrine carcinoma with multiple liver metastases and diabetes mellitus. The patient received chemotherapies from 2011 to 2018 along with radiation therapy in 2017 for bone metastases. On the second day of hospitalization, fever and chills began, accompanied by watery stool and abdominal pain. Her complete blood counts were as follows: leukocytes, $5.2 \times 10^{9} / \mathrm{L}$ (neutrophil $82.9 \%$ ); hemoglobin, $88 \mathrm{~g} / \mathrm{L}$; platelets, $111 \times 10^{9} / \mathrm{L}$. Results of biochemical analysis were as follows: BUN/Cr, 15.7/0.15 nmol/L; C-reactive protein, 1,625.7 $\mathrm{nmol} / \mathrm{L}$. Urinalysis with microscopy showed 2+ blood, 2+ ketone,
Received: October 10, 2019

Revision received: December 6, 2019

Accepted: January 28, 2020

Corresponding author: Daewon Kim, M.D.

Department of Laboratory Medicine and Research Institute of Bacterial Resistance, Yonsei University College of Medicine, 50-1 Yonsei-ro, Seodaemun-gu, Seoul 03722, Korea

Tel: +82-10-3340-3471, Fax: +82-2-2227-8353, E-mail: fseraph85@yuhs.ac

Co-corresponding author: Dongeun Yong, M.D., Ph.D.

Department of Laboratory Medicine and Research Institute of Bacterial Resistance, Yonsei University College of Medicine, 50-1 Yonsei-ro,

Seodaemun-gu, Seoul 03722, Korea

Tel: +82-2-2228-2442, Fax: +82-2-313-0956, E-mail: deyong@yuhs.ac

\section{(c) (1) $(9$}

(C) Korean Society for Laboratory Medicine

This is an Open Access article distributed under the terms of the Creative Commons Attribution Non-Commercial License (https://creativecommons.org/licenses/by-nc/4.0) which permits unrestricted non-commercial use, distribution, and reproduction in any medium, provided the original work is properly cited. 
Table 1. Recent worldwide reports of non- $\mathrm{H}_{2} \mathrm{~S}$-producing Salmonella isolated from clinical samples

\begin{tabular}{lccl}
\hline Reported year & Country & Cases (N) & \multicolumn{1}{c}{ Serovar } \\
\hline $2014[4]$ & Kuwait & 1 & Kentucky \\
$2015[5]$ & China & 19 & Choleraesuis \\
$2016[6,7,8]$ & China & 37 & Senftenberg (30), Aberdeen (7) \\
$2017[9]$ & United States & 1 & Cerro \\
$2018[10]$ & China & 46 & $\begin{array}{l}\text { Gallinarum (15), Typhimurium (8), } \\
\text { Choleraesuis (6), Paratyphi A (6), } \\
\end{array}$ \\
& & & $\begin{array}{l}\text { Meleagridis (2), Agona (2), } \\
\text { Thompson (2), Enteritidis (1), } \\
\end{array}$ \\
& & & $\begin{array}{l}\text { Derby (1), Paratyphi B (1), Hadar } \\
\text { (1), Give (1) }\end{array}$ \\
2020 & & & Infantis
\end{tabular}

and 3+ leukocyte esterase (scale of 0 to $4+$ with urine strip), along with a white blood cell count $>30 /$ high-power field, indicating pyuria. Notably, two blood cultures and a urine culture obtained on the second and third days of hospitalization, respectively, showed no growth, whereas PCR analysis of stool sample was positive for Salmonella. Abdominopelvic computed tomography showed no bowel wall thickening. On the fourth day, a stool culture was obtained and no colony was detected.

Two sets of blood culture bottles (BacT/Alert FA Plus and BacT/ Alert FN Plus; bioMérieux, Marcy-l'Étoile, France) were obtained on the fourth day and immediately incubated in a BacT/Alert 3D automated blood culture system (bioMérieux). After 24 hours, a specific colorimetric visual signal was detected in the BacT/Alert FN plus bottle. Subculture on MacConkey agar plate showed colorless, pale colonies of gram-negative rods (Fig. 1A). Matrixassisted laser desorption ionization-time-of-flight mass spectrometry (MALDI-TOF MS) using VITEK MS (bioMérieux) and Bruker Biotyper (Bruker Daltonics, Bremen, Germany) identified Salmonella species (score 22.0 ) with $99 \%$ reliability. The isolate was identified as belonging to serogroup $C$ with a polyvalent antisera kit (JoongKyeom, IIsan, Korea). The TSI agar biochemical test results were as follows: alkaline, acid, gas-negative, $\mathrm{H}_{2} \mathrm{~S}$-negative (Fig. 1B). The isolate was sent to Research Institute of Public Health and the Environment, Seoul, Korea, to further identify the serotype, and Salmonella enterica serovar Infantis was confirmed.

Anti-microbial susceptibility of the isolate was determined by the automated broth microdilution method using VITEK 2 (bioMérieux). The isolate was resistant to cefazolin (minimal inhibitory concentration $[\mathrm{MIC}], 4 \mu \mathrm{g} / \mathrm{mL}$ ), cefoxitin (MIC, $4 \mu \mathrm{g} / \mathrm{mL}$ ), amikacin (MIC, $2 \mu \mathrm{g} / \mathrm{mL}$ ), and gentamicin (MIC, $1 \mu \mathrm{g} / \mathrm{mL}$ ). The

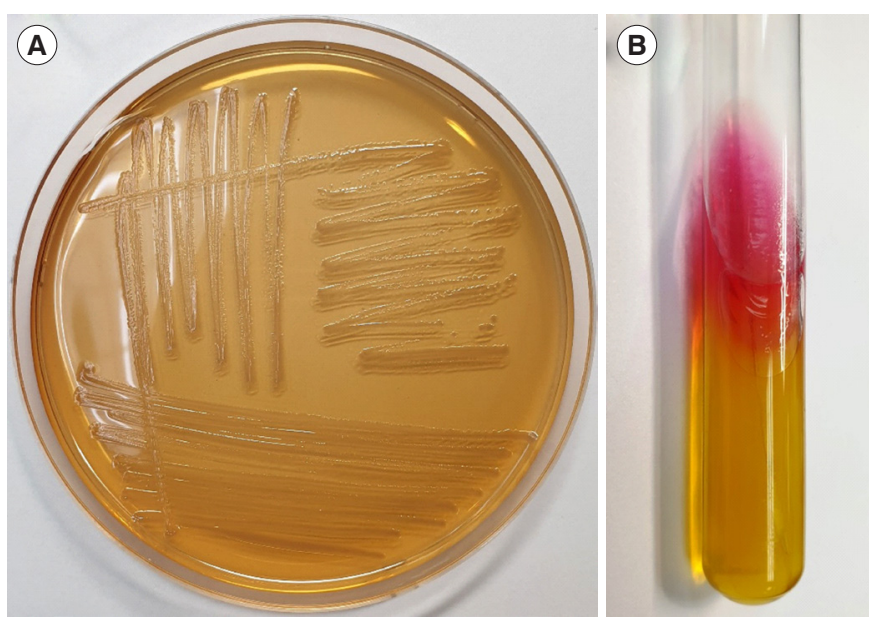

Fig. 1. Biochemical result of non- $\mathrm{H}_{2} \mathrm{~S}$-producing Salmonella (A) Pale and colorless colonies on a MacConkey agar plate. (B) Non$\mathrm{H}_{2} \mathrm{~S}$-producing bacteria in triple sugar iron agar.

patient was empirically treated with piperacillin/tazobactam and levofloxacin due to suspected urinary tract or gastrointestinal infection. Metronidazole and ciprofloxacin were administered based on the positive Salmonella PCR result. The blood culture on the eighth day of hospitalization was negative. However, ceftriaxone was administered because fever spiking persisted until discharge (February 2019).

Direct sequencing of the phs and cysJlH operons of the isolate was performed, and the sequences aligned to those of strain Salmonella enterica serovar Infantis FSIS1502916 (NZ_CP016408.1) using DNAman 6.0 (Lynnon, Quebec, Canada). The primers were as follows: cysJ1 forward 5'-CGGCATCGGACAGTTTACCT-3' and reverse 5'-CTGGATGCTCAGGCGCTAAT-3', cysJ2 forward 5'-GATTAGCGCCTGAGCATCCA-3' and reverse 5'-CGTGTCGATGCTGACGTTGA-3', cysJ3 forward 5'-TTCAACGTCAGCATCGACAC-3' and reverse 5'-CTGCGTTTACTCACCCCAGT-3', cysI1 forward 5'-CTCGCGGTATACCTTGCCTG-3' and reverse 5'-CTGCTGGAGATCGCGAAGAT-3', cysI2 forward 5'-GCACGCTGGCAATAATCAGG-3' and reverse 5'-TGCATGCTGAAGCCTACGAA-3', cysl3 forward 5'-CGATATCATTTTGCGGCGGG-3' and reverse 5'-CGGTATGGACCTCGAATCGG-3', cysH forward 5'-GCCGGATTTTTCATAGCAGCA-3' and reverse 5'-CAGGCAAGGTATACCGCGAG-3', and previously reported primers for phs [10]. Unlike previous cases [10], no point mutation was found in the coding region of the phs operon, whereas a nucleotide change (C to T) at the first base pair in the 3'-untranslated region of phsC was observed. In addition, a 1247A>G variant, causing the V416A missense mutation, was found in cysJ. This is the first report of a missense mutation in the cys gene in non- $\mathrm{H}_{2} \mathrm{~S}$-producing Sal- 
monella. However, further research is needed to determine whether these mutations influence $\mathrm{H}_{2} \mathrm{~S}$ production or not.

Since the identification of Salmonella serotype is not possible in most clinical laboratories using conventional biochemical testing, non- $\mathrm{H}_{2} \mathrm{~S}$-producing Salmonella can be misdiagnosed as Salmonella Typhi or even other Enterobacteriaceae. Therefore, caution is needed with the diagnosis of Salmonella infection.

\section{ACKNOWLEDGEMENTS}

We are grateful to researchers of Research Institute of Bacterial Resistance for excellent technical assistance.

\section{AUTHOR CONTRIBUTIONS}

KSL, DK and DY designed the study and secured the funding. KSL performed the experiments. KSL and DK analyzed and interpreted the data and wrote the manuscript. DY, HL and $\mathrm{KL}$ revised the manuscript draft.

\section{CONFLICT OF INTEREST}

No potential conflicts of interest relevant to this article were reported.

\section{RESEARCH FUNDING}

This work was supported by a grant from the Korea Health Technology R\&D Project through the Korea Health Industry Development Institute (KHIDI), funded by the Ministry of Health \& Welfare, Republic of Korea (grant number: HI14C1324); by the Research Program funded by the Korea Centers for Disease Control and Prevention, Ministry of Health and Welfare, Republic of Korea(2019-ER5403-00); by Korea Institute of Planning and Evaluation for Technology in Food, Agriculture, Forestry and Fisheries (IPET) through Agricultural Microbiome R\&D Program. The funders had no role in the study design, data collection and analysis, decision to publish, or preparation of the manuscript.

\section{ORCID}

Kwang Seob Lee

https://orcid.org/0000-0002-6286-108X

Daewon Kim

Hyukmin Lee

Kyungwon Lee

Dongeun Yong https://orcid.org/0000-0002-8523-4126

https://orcid.org/0000-0003-3788-2134

https://orcid.org/0000-0002-1225-8477

\section{REFERENCES}

1. Infectious disease portal, Korea center for disease control \& prevention, http://www.cdc.go.kr/npt/biz/npp/iss/gastrointestinalStatisticsMain.do (Updated on Jan 2020)

2. Chen HM, Wang Y, Su LH, Chiu CH. Nontyphoid Salmonella infection: microbiology, clinical features, and antimicrobial therapy. Pediatr Neonatol 2013;54:147-52.

3. Sakano C, Kuroda M, Sekizuka T, Ishioka T, Morita Y, Ryo A, et al. Genetic analysis of non-hydrogen sulfide-producing Salmonella enterica serovar Typhimurium and S. enterica serovar Infantis isolates in Japan. J Clin Microbiol 2013;51:328-30.

4. Albert MJ, Al Obaid K, Alfouzan W, Sheikh AR, Udo E, Izumiya H, et al. Isolation of Salmonella enterica serovar Kentucky strain ST 198 and its $\mathrm{H}_{2} \mathrm{~S}$-negative variant from a patient: implications for diagnosis. J Clin Microbiol 2014;52:4090-3.

5. Xie J, Yi S, Zhu J, Li P, Liang B, Li H, et al. Antimicrobial resistance and molecular investigation of $\mathrm{H}_{2} \mathrm{~S}$-negative Salmonella enterica subsp. enterica serovar Choleraesuis isolates in China. PLoS One 2015;10:e0139115.

6. Abd El Ghany M, Shi X, Li Y, Ansari HR, Hill-Cawthorne GA, Ho YS, et al. Genomic and phenotypic analyses reveal the emergence of an atypical Salmonella enterica serovar Senftenberg variant in China. J Clin Microbiol 2016;54:2014-22.

7. Yi S, Xie J, Liu N, Li P, Xu X, Li H, et al. Emergence and prevalence of non- $\mathrm{H}_{2} \mathrm{~S}$-producing Salmonella enterica serovar Senftenberg isolates belonging to novel sequence type 1751 in China. J Clin Microbiol 2014; 52:2557-65

8. Wu F, Xu X, Xie J, Yi S, Wang J, Yang X, et al. Molecular characterization of Salmonella enterica serovar Aberdeen negative for $\mathrm{H}_{2} \mathrm{~S}$ production in China. PLoS One 2016;11:e0161352.

9. Kovac J, Cummings KJ, Rodriguez-Rivera LD, Carroll LM, Thachil A, Wiedmann M. Temporal genomic phylogeny reconstruction indicates a geospatial transmission path of Salmonella Cerro in the United States and a clade-specific loss of hydrogen sulfide production. Front Microbiol 2017;8:737

10. Xie J, Wu F, Xu X, Yang X, Zhao R, Ma Q, et al. Antibiotic resistance and molecular characterization of the hydrogen sulfide-negative phenotype among diverse Salmonella serovars in China. BMC Infect Dis 2018;18: 292. 